\title{
In Defense of a Developmental Theory of Intelligence: Response to the Commentators
}

\author{
Andreas Demetriou $^{a}$ Nikolaos Makris ${ }^{b}$ George Spanoudis ${ }^{c}$ \\ a University of Nicosia, Nicosia, Cyprus; ${ }^{b}$ Democritus University of Thrace, Thrace, Greece; \\ 'University of Cyprus, Nicosia, Cyprus
}

Response to Commentaries by Philip Zelazo; Ulrich Müller and Mauricio Garcia-Barrera on A. Demetriou et al.: "Mapping the Dimensions of General Intelligence: An Integrated Differential-Developmental Theory" [Human Development 2018; 61: 4-42]

We are honored by our commentators, Ulrich Müller and Mauricio Garcia-Barrera [2018] and Philip David Zelazo [2018], for their commentaries on our paper which summarizes and updates our developmental theory of intelligence [Demetriou, Makris, Kazi, Spanoudis, Shayer, \& Kazali, 2018]. This theory, drawing on a long series of empirical studies (see references in the target article) and three powerful research traditions focusing on the human mind, aims to account for three aspects of the human mind: the mental processes involved in real-time understanding and problem solving (cognitive tradition [Baddelley, 2012; Johnson-Laird, 2012]), their development and interactions from birth to adulthood (developmental tradition [Case, 1985; Pascual-Leone, 1988; Piaget, 1970; Zelazo, 2015]), and the factors causing individual differences in the state and development of mental processes (differential tradition [Carroll, 1993; Jensen, 1998; Spearman, 1927]). However, it is noted that the theory is broader than what is presented in the target paper. The target paper focuses on the dimensions of general intelligence, specifying their organization and development. In its fullness, the theory also specifies mind-personality relations, the brain bases of the processes involved, their grounding into culture, and outlines its implications for education. For a complete presentation, the reader is referred to our recent book [Demetriou \& Spanoudis, 2018]. This book answers many of the concerns of the commentators but it was not available when the target paper was published. Here we focus on the points raised by the two commentaries.

\section{Balancing between the Sin of Generality and the Temptation of Specificity}

General theories are always vulnerable to the specificity criticism: the more abstract they become as they expand their realm of application, the more they may fail to account for specificities in the domains involved. This is a universal epistemological problem. Newton's theory of gravity obviously accounts better for planetary motion than for the movement of a falling leaf.

\begin{tabular}{llll}
\hline KARGER & & Andreas Demetriou \\
E-Mail karger@karger.com & & University of Nicosia \\
www.karger.com/hde & & Makedonitissis 46 \\
& CY-2417 Nicosia (Cyprus) \\
& E-Mail ademetriou @ ucy.ac.cy
\end{tabular}


Einstein's theory of relativity accounts better than Newton's theory for huge masses at the cosmic scale but it is not useful for building earthquake-resistant buildings. Civil engineers do not need either of these grand theories to design durable houses, although gravity is an important factor to be dealt with in construction. Natural scientists are more at ease with the generality-specificity question because they know that both generality and specificity come with a cost: The more general a theory becomes the more distant it may be from accounting for specific realities; the more specific it becomes to a domain the more distant it may be from other domains. In psychology, we are ambivalent about general theories. We complain when we do not have them but we also complain when they come.

In this spirit, Zelazo [2018] notes that the theory is vulnerable to criticisms that have plagued other grand theories, insofar as it "relies on abstractly specified, domain general developmental mechanisms." He suggests that the theory neglects (a) the role of personality in cognitive functioning, (b) how specific cognitive developmental processes are instantiated in the brain, and (c) the influence of culture and specific social interactions.

An epistemological approach to these criticisms would ask: Does a cognitive developmental theory need to account for any of these realities? Not necessarily, at the present epistemological state of the field. A good cognitive developmental theory may speak with a good personality theory, as long as each presents a sound and solid account for its own domain. Also, it does not need to specify how the mental processes it describes are instantiated in the brain as long as it is precise enough to direct brain research to look for these processes in the brain. In fact, a problem of present brain research is that there is no generally acceptable cognitive theory to guide brain scientists toward what to study in the brain [see Haier, 2017]. Along the same line, a good developmentaldifferential theory of the mind may come up with good theories about how culture and society influence humans to map and explicate the cultural-social causes of variations along its dimensions of developmental and individual variation.

Of course, unification is a long-term dream for any science. For instance, physics strives for the theory of everything that would account for the natural world at all scales, from subatomic particles to the cosmos. The theory of everything in biology would account for all phenomena of life, from genes to species across all times. In pursuit of the dream, we did make steps in the direction of integrating the theory with all three realms noted by Zelazo. With regard to personality and motivation, a full chapter in the book [Demetriou \& Spanoudis, 2018] draws the connections between cognitive development and personality development. We explicate how cognizance, one of the central mechanisms of cognitive change, interlinks cognitive development with personality development. With increasing refinement and resolution of cognizance, developing persons improve in their ability to regulate themselves and choose environments suited to their dispositions. In a series of recent studies, we show how mind and personality interact in development. For instance, we show that some aspects of personality impede (conscientiousness) and some other aspects accelerate cognitive development (extroversion and openness to experience), especially at transitions between cycles [Demetriou, Spanoudis, Zebec, Andreou, Golino, \& Kazi, in press].

We do not have brain research of our own. However, we did draw the connections between the general premises of the theory and current brain research. A full chapter in the book discusses these connections. In summary, we argue that there are several (overlapping) networks in the brain related to each one of the mental functions specified in the theory (e.g., there are sensory networks associated with core processes underlying psychological domains; frontal and hippocampal regions related to working memory; temporal, parietal, and prefrontal networks related to integrative reasoning processes; frontal and medial networks related to monitoring, awareness, and regulation). Also, brain research suggests that different mental processes associate with different brain rhythms. For instance, specific representations in working memory associate with high frequency rhythms (i.e., beta and gamma) and integrative processes, such as reasoning and awareness, associate with lower frequency rhythms (i.e., delta and theta). Changes in the synchronization between these rhythms relate to changes in the cycles of cognitive change described by the theory. Finally, differences in intelligence relate to differences in the refinement and activation efficiency of brain networks related to reasoning and problem solving. We offer these assumptions as a research program rather than a theory that might bring cognitive-developmental science and brain science closer to each other.

In Defense of a Developmental Theory of Intelligence
Human Development 2018;61:138-143 DOI: $10.1159 / 000489840$ 
We do have cross-cultural research on various aspects of the theory [see Demetriou \& Spanoudis, 2018]. This research suggests that the organization and development of the mind as specified by the theory is the same across human cultures. We argue that nothing else would have to be expected: the human mind is framed by the same genome and it is implemented by the same brain. Even the overall conception of the human mind is similar across cultures because thinkers in all cultures see the similarities, regardless of place. Of course, cultural differences do exist in important cultural institutions affecting learning and problem solving, such as language and writing systems. These differences are expressed in differences in intellectual attainment related to these cultural-institutional differences, as they interact with the constructs involved. For instance, we showed that learning the logographic Chinese system of reading and writing massively trains processing and representational process, especially in spatial reasoning. As a result, Chinese develop faster in this domain but not in other domains, such as deductive reasoning. This may be associated with the fact that Chinese (and other cultures using similar reading and writing systems) exceed Western cultures in IQ [Demetriou, Zhang, Spanoudis, Christou, Kyriakides, \& Platsidou, 2005; Kazi, Demetriou, Spanoudis, Zhang, \& Wang, 2012].

\section{Is g Equal to Executive Function?}

Müller and Garcia-Barrera [2018] raise several important issues to be clarified. A focal point of their criticism is that we do not consistently deal with executive function, sometimes decomposing it into distinct components, such as attention control, flexibility, and working memory, and sometimes speaking about an underlying common executive function permeating all these components. We think that Müller and Garcia-Barrera create a straw man to have the pleasure of killing it. Our formula, which states that $\mathrm{g}=\mathrm{f}$ (attention control + flexibility + working memory + cognizance + inference), is shorthand for a very consistent finding of our structural equation models: Variance in $g$ abstracted from performance on several domains of reasoning is distinctly decomposed into these factors, each of which is represented in the model by specifically designed tasks operationalizing the respective construct (see Fig. 1 in the target article). The addition sign " + " indicates that they are separate but combinable. Admittedly, something minimally goes through all of them but each of them uses it differently to serve different task-specific behavioral needs. The more complex the task the more is needed from what is involved in each of these factors. In fact, we noted elsewhere that interactive or mutualist models assuming that $g$ resides in the interactions of these processes rather than in any single process along may be a good description of g [van der Maas, Kern, Marsman, \& Stevenson, 2017], once we recognize that taskspecific interactions are not blind. They have a subjective aspect that makes them personally valued representations and choices rather than successful engineering solutions as it occurs in AI programs or creatures, such as robots. Cognizance is the mechanism of subjectivity. Attention may be unconscious to the extent that it is captured by salient stimuli and it may become conscious when it is systematically refocused according to a preset represented task intentionally brought to bear on the salient stimulus. In conclusion: $g$ is not equal to executive function; executive function includes several processes, all differentially needed by complex tasks.

\section{Differentiation and Infinite Regress}

Müller and Garcia-Barrera [2018] also claim that we fail to address how executive function is integrated with general ability in development and how it possibly differentiates with age. This is definitely not the case. Based on a long series of studies covering the whole lifespan, we recently showed how mental processes, including all processes in the formula above, intertwine with and differentiate from $g$ in each developmental cycle [Demetriou et al., 2017]. We showed that differentiation/de-differentiation of specific processes from $g$ is a developmental rather than an individual differences phenomenon. Thus, the relation between specific processes and g varies with developmental cycle and phase. New acquisitions in each cycle get increasingly integrated 
into g, infusing it with their properties. Control of attentional focus, awareness of the perceptual origins of knowledge, awareness of inferential processes, and precise self-evaluation dominate in $\mathrm{g}$ at 2-4, 4-6, 8-10, and 13-14 years, respectively. Once serving their developmental role, they subsequently part company with $\mathrm{g}$. Thus, executive functions remorph in development.

Müller and Garcia-Barrera [2018] also criticize the fact that EF and cognizance appear as processes at two different levels, being constituents of both $g$ and the Abstraction, Alignment, Cognizance (AACog) mechanism orchestrating the interactions between the processes constituting g. They claim that this renders the theory culpable of infinite regress because the same processes (EF and cognizance) appear to be both regulators and being regulated. AACog is the representational part of g, fusing together (a) the mapping of representations onto each other according to a goal - which is itself another representation (alignment), (b) abstraction of commonalities between them (inference), and (c) their evaluation and encoding into new representations (cognizance). Thus, the formula above may be re-written as follows: $g=f$ (attention control + working memory + AACog).

This implies that flexibility (a term used in the literature for some shifting tasks [Zelazo, 2015]), inference (all sorts of reasoning), and cognizance are packed into the AACog mechanism. EF requires cognizance (minimal awareness of a goal and at least one action to attain it) but they are not identical. Cognizance is broader, additionally involving awareness of mental processes involved, past experiences, etc. Thus, cognizance is needed by the regulatory component of executive control as much as executive control, in its implementation, generates experiences registered by cognizance. One might see infinite regress in a static system; however, the mind is not static: it develops in cycles where what is under regulation now (plans in sake of a goal) improves (regulates) the regulatory mechanism itself (cognizance) in successive cycles of differentiation and de-differentiation. In a sense, the processes involved are not the same across cycles. In fact, this is what the theory is about.

Having advanced the argument above, we are willing to agree with our commentators that some cleaning and clarification is needed in the field. The confounding and overlap between these constructs is due, to some extent, to the fact that they are used in each of the different traditions noted above under different behavioral and experimental contexts. We hope that both, the target article and the commentaries will inspire further research specifically designed to operationalize and unconfound them.

\section{Structure versus Function}

Müller and Garcia-Barrera [2018] note that the theory is mainly about architecture rather than function and it thus needs to be supplemented by a functional framework. Under some conditions, we would agree with this point. The conditions are as follows: First, it must be kept in mind that this is a developmental theory. Therefore, the representational profile of each cycle is a frame of functional possibilities. It defines what can be represented, understood, and learned, and how this may be done in each cycle. Second, it specifies a mechanism of transition across cycles, cognizance, that constrains interactions with the world and ensuing learning. In support of this claim, we presented a series of studies showing that training this mechanism causes transitions in other domains, such as deductive and mathematical reasoning [Christoforides, Spanoudis, \& Demetriou, 2016; Papageorgiou, Christou, Spanoudis, \& Demetriou, 2016]. Under these conditions, the theory needs to develop in the direction of specifying precise functional mechanisms of learning and development at successive developmental cycles. Probabilistic models of learning [see Tourmen, 2016], including Bayesian learning [Tenenbaum, Kemp, Griffiths, \& Goodman, 2011] may be part of the answer. Integrating these models in the theory might highlight how representations emerge, initially, from interactions with the world, especially during the cycles of the episodic (birth to 2 years) and realistic representational mind (2-6 years). Indeed, deep neural networks succeeded, in recent years, in solving formidable tasks in several domains, sometimes beating humans. But still, these models would have to account for the increasing compositionality and creativity of the human mind, that these artificial systems do not have despite their formidable success in their circumscribed domains of application. Interestingly, experts in the field

In Defense of a Developmental Theory of Intelligence
Human Development 2018;61:138-143 DOI: $10.1159 / 000489840$ 
of human-like artificial intelligence accept that learning-to-learn is needed, if this is to be possible [Lake et al., 2017]. We hope that the readers, including our commentators, recognize that a large part of our theory is about learning-to-learn. Thus, the theory does have much to gain from this new field; hopefully, it also has much to offer to it, in specifying types of mind that may be simulated and transitions from one type to the other.

\section{How Mentalist Are Infants?}

Finally, Müller and Garcia-Barrera [2018] claim that we are not explicit enough regarding the mental possibilities of infants: i.e., if we consider infants mentalistic creatures in the neonativist sense of crediting infants with awareness of mental states or in a more lenient sense of emerging awareness, starting from a state where awareness involves the objects of thought but not mental processes or states themselves. They warn us that subscribing to the first view would make the theory vulnerable to the criticisms plaguing empiricist representational theory that it cannot account for how representations emerge in the first place. We hoped that we were clear that cognizance emerges and becomes increasingly refined and resolved at several levels, starting from not present at all, up to 1-2 months of age, to becoming action-based at circa the first year, to registering bodily characteristics and associate with intentions of others at the end of the second year of life, etc. Thus, cognizance and awareness in our theory is an interactive agent, bridging the individual with the world and ensuring the subjectivity and meaning of one's own experience.

\section{Conclusion}

We fully concur with Zelazo that the theory echoes the consensus emerging in the sciences of the human mind over more than a century of scholarship about the key characteristics of the organization and development of the human mind. We also trust that the issues raised by the two commentaries and the discussion that is starting will further expand our understanding of the developing mind.

\section{References}

Baddeley, A.D. (2012). Working memory: Theories, models, and controversies. Annual Review of Psychology, 63, 1-29. doi:10.1146/annurev-psych-120710-100422

Carroll, J.B. (1993). Human cognitive abilities: A survey of factor-analytic studies. New York, NY: Cambridge University Press. doi:10.1017/CBO9780511571312

Case, R. (1985). Intellectual development. Birth to adulthood. New York, NY: Academic Press.

Christoforides, M., Spanoudis, G., Demetriou, A. (2016). Coping with logical fallacies: A developmental training program for learning to reason. Child Development, 87, 1856-1876. doi:10.1111/cdev.12557

Demetriou, A., Makris, N., Kazi, S., Spanoudis, G., Shayer, M., \& Kazali, E. (2018). Mapping the dimensions of general intelligence: An integrated differential-developmental theory. Human Development, 61, 4-41. doi: $10.1159 \% 2 \mathrm{~F} 000484450$

Demetriou, A., \& Spanoudis, G. (2018). Growing minds: A general theory of intelligence and learning. London, UK: Routledge.

Demetriou, A., Spanoudis, G., Kazi, S., Mouyi, A., Žebec., M.S., Kazali, E., Golino, H. F., Bakracevic, K., \& Shayer, M. (2017). Developmental differentiation and binding of mental processes with re-morphing g through the life-span. Journal of Intelligence, 5, 23. doi:10.3390/jintelligence5020023

Demetriou, A., Spanoudis, G., Zebec, M., Andreou, M., Golino, H., \& Kazi, S. (in press). Mind personality relations from childhood to adolescence. Journal of Intelligence.

Demetriou, A., Zhang, X.K., Spanoudis, G., Christou, C., Kyriakides, L., \& Platsidou, M. (2005). The architecture and development of mental processing: Greek, Chinese or Universal? Intelligence, 33, 109-141. doi:10.1016/j.intell.2004.10.003

Haier, R.J. (2017). The neuroscience of intelligence. Cambridge, UK: Cambridge University Press.

Jensen, A.R (1998). The g factor: The science of mental ability. Westport, CT: Praeger. 
Johnson-Laird, P.N. (2012). Inference with mental models. In K.J. Holyoak \& R.G. Morrison (Eds.), The Oxford handbook of thinking and reasoning (pp. 134-145). New York, NY: Oxford University Press. doi:10.1093/oxfordhb/9780199734689.013.0009

Kazi, S., Demetriou, A., Spanoudis, G., Zhang, X.K., \& Wang, Y. (2012). Mind-culture interactions: How writing molds mental fluidity. Intelligence, 40, 622-637. doi:10.1016/j.intell.2012.07.001

Lake, B.M., Ullman, T.D., Tenenbaum, J.B., \& Gershman, S.J. (2017). Building machines that learn and think like people. Behavioral and Brain Sciences. doi:10.1017/S0140525X16001837

Müller, U., \& Garcia-Barrera, M. (2018). Developmental g: Lost in the construct jungle. Commentary on Demetriou. Human Development. doi:10.1159/000486750

Papageorgiou, E., Christou, C., Spanoudis, G., \& Demetriou, A. (2016). Augmenting intelligence: Developmental limits to learning-based cognitive change. Intelligence, 56, 16-27. doi:10.1016/j.intell.2016.02.005

Pascual-Leone, J. (1988). Organismic processes for neo-Piagetian theories: A dialectical causal account of cognitive development. In A. Demetriou (Ed.), The neo-Piagetian theories of cognitive development: Toward an integration (pp. 25-64). Amsterdam: North-Holland.

Piaget, J. (1970). Piaget's theory. In P.H. Mussen (Ed.), Carmichael's handbook of child development (pp. 703-732). New York, NY: Wiley.

Spearman, C. (1927). The abilities of man: Their nature and measurement. London: MacMillan.

Tenenbaum, J.B., Kemp, C., Griffiths, T.L., \& Goodman, N.D. (2011). How to grow a mind: Statistics, structure, and abstraction. Science, 331, 1279-1285. doi:0.1126/science.1192788

Tourmen, C. (2016). With or beyond Piaget? A dialogue between new probabilistic models of learning and the theories of Jean Piaget. Human Development, 59, 4-25. doi:10.1159/000446670

van der Maas, H.L.J., Kan, K., Marsman, M., \& Stevenson, C.E. (2017). Network models for cognitive development and intelligence. Journal of Intelligence, 5, 16. doi:10.3390/jintelligence5020016

Zelazo, P.D. (2015). Executive function: Reflection, iterative reprocessing, complexity, and the developing brain. Developmental Review, 38, 55-68. doi:10.1016/j.dr.2015.07.001

Zelazo, P.D. (2018). Abstracting and aligning essential features of cognitive development. Human Development. doi:10.1159/000486749

In Defense of a Developmental Theory of

Human Development 2018;61:138-143 